\title{
Characterization of the Bayes Estimator and the MDL Estimator for Exponential Families
}

\author{
Jun-ichi Takeuchi
}

\begin{abstract}
We analyze the relationship between a Minimum Description Length (MDL) estimator (posterior mode) and a Bayes estimator for exponential families. We show the following results concerning these estimators: a) Both the Bayes estimator with Jeffreys prior and the MDL estimator with the uniform prior with respect to the expectation parameter are nearly equivalent to a bias-corrected maximum-likelihood estimator with respect to the canonical parameter. b) Both the Bayes estimator with the uniform prior with respect to the canonical parameter and the MDL estimator with Jeffreys prior are nearly equivalent to the maximum-likelihood estimator (MLE), which is unbiased with respect to the expectation parameter. These results together suggest a striking symmetry between the two estimators, since the canonical and the expectation parameters of an exponential family form a dual pair from the point of view of information geometry. Moreover, a) implies that we can approximate a Bayes estimator with Jeffreys prior simply by deriving an appropriate MDL estimator or an appropriate bias-corrected MLE. This is important because a Bayes mixture density with Jeffreys prior is known to be maximin in universal coding [7].
\end{abstract}

Index Terms-Bayes estimator, exponential family, higher order asymptotic theory, information geometry, Minimum Description Length principle, universal source coding.

\section{INTRODUCTION}

I $\mathrm{N}$ this study, we examine the estimation of parameters of probability densities in the general class of regular exponential families [5]. In particular, we analyze the relationship between the following two estimators and reveal a symmetry between the two: a Minimum Description Length (MDL) estimator [12] (also called a Minimum Message Length estimator [15], [16], which has a posterior mode interpretation) and the estimator which is Bayes with respect to Kullback-Leibler divergence (KL divergence for short) between the parameterized densities (it can be obtained by projecting the Bayes posterior mixture density onto the original exponential family).

In the field of universal source coding, Bayes mixture densities have recently become a popular subject of study for two main reasons: 1) Bayes decision theory can be used to determine the code which will achieve the minimax redundancy [7]-[9], [11], and 2) codes based on a Bayes mixture will be superior to two-step codes [13]. The MDL estimator we study here is based on a two-step code (This is one form

Manuscript received July 27, 1995; revised October 12, 1996. The material in this paper was presented at the IEEE International Symposium on Information Theory, Whistler, BC, Canada, September 18-22, 1995.

The author is with the Theory NEC Laboratory, RWCP (Real World Computing Partnership) c/o C\&C Research Laboratories, NEC Corporation. 4-1-1 Miyazaki, Miyamae-ku, Kawasaki, Kanagawa 216, Japan.

Publisher Item Identifier S 0018-9448(97)03780-2. of Rissanen's MDL principle [12], which more generally also encompasses mixture-based codes).

In two-step coding, we first use observed data to estimate a source distribution, which we then encode. Next, on the basis of the estimate, we encode the observed data. In the MDL principle, an optimal estimate is defined as that which gives the shortest total code length for given data, and we refer to estimators based on the MDL principle as MDL estimators. MDL estimators have been shown to be especially effective when the complexity (i.e., number of parameters) of the actual source distribution is unknown [2], [3], [18], [19]. (Here, however, we will focus on the case of parameter estimation in a fixed family.)

By way of contrast, Bayes codes do not encode the source distribution and are a fundamentally different from the twostep type. Consider a parametric family of probability densities, $S=\{p(x \mid \theta) \mid \theta \in \Theta\}$ on a space $\mathcal{X}$, and a predictive density estimator $f\left(x_{t+1} \mid x^{t}\right)$ for $S$ (i.e., $\sum_{x \in \mathcal{X}} f\left(x \mid x^{t}\right)=1$ ). The cumulative risk with KL divergence is defined as

$$
R_{N}(\theta, f) \equiv \sum_{t=1}^{N} \int p\left(x^{t} \mid \theta\right) \ln \left(p\left(x_{t} \mid \theta\right) / f\left(x_{t} \mid x^{t-1}\right)\right) d x^{t}
$$

where "ln" denotes the natural logarithm,

$$
p\left(x^{t} \mid \theta\right)=\prod_{u=1}^{t} p\left(x_{u} \mid \theta\right)
$$

and $\theta$ is the actual parameter. This cumulative risk is also the redundancy of the code based on the joint density

$$
f\left(x^{N}\right)=\prod_{t=0}^{N-1} f\left(x_{t+1} \mid x^{t}\right)
$$

(see [6]). Next, the Bayes risk (or Bayes redundancy) of $f$ with respect to the prior $w(\theta) d \theta$ is defined as

$$
R_{N}(w, f) \equiv \int R_{N}(\theta, f) w(\theta) d \theta
$$

The Bayes predictor with this prior uses

$$
f_{w}\left(x_{t+1} \mid x^{t}\right) \equiv \int p(x \mid \theta) w\left(\theta \mid x^{N}\right) d \theta
$$

which achieves $\min _{f} R_{N}(w, f)$ (where $w\left(\theta \mid x^{N}\right)$ is the posterior density).

The Bayes predictor with the Jeffreys prior [10] (denoted by $\left.w_{J}\right)$ is of special importance among the Bayes procedures, since $f_{w_{J}}$ asymptotically maximin for the redundancy 
$R_{N}(\theta, f)$ and modifications of it are asymptotically minimax under certain conditions [7], [17], [4].

For Bernoulli sources $(\mathcal{X}=\{0,1\}, p(1 \mid r)=r)$, we have

$$
f_{w_{J}}\left(1 \mid x^{N}\right)=(k+0.5) /(N+1)
$$

( $k$ is the number of occurrences of " 1 " in $N$ trials), i.e., a classic Laplace estimator is maximin for the Bayes redundancy. Moreover, this estimator can be derived from the MDL principle combined with the assumption that we either use the binary digit expansion of the value of $r$ itself as the code for the source distribution or assume that the prior is uniform over the range of $r$ [20], [16]. Bayes procedures for coding (unlike the two-step code) necessarily involve mixtures and the corresponding predictive density estimates suffer from the problem that, in general, they do not belong to the class of the original source. When an estimate belongs to the original class (i.e., densities estimated by plugging in parameter estimators), we say that the estimator is "proper." Bayes predictors are not always proper, while MDL estimators are: An MDL estimate (based on a two-step code) always belongs to the original class.

Inspired by the circumstances mentioned above, we analyze the relationship between $f_{w}$ and the MDL estimator for general exponential families. Since we do not have the means to analyze the relationship between them directly, we consider the proper estimator $f$ minimizing $R_{N}(w, f)$. We let $\tilde{f}_{w}\left(\cdot \mid x^{N}\right)=$ $p\left(\cdot \mid \hat{\theta}_{w}\right)$ denote such an estimator. Note that $\hat{\theta}_{w}$ is equal to

$$
\arg \min _{\theta^{\prime}} \int D\left(p(\cdot \mid \theta) \| p\left(\cdot \mid \theta^{\prime}\right)\right) w\left(\theta \mid x^{N}\right) d \theta .
$$

(We let $D(p \| q)$ denote KL divergence of $q$ with respect to p.) By simple manipulation, we can see $\tilde{f}_{w}\left(\cdot \mid x^{N}\right)=p\left(\cdot \mid \hat{\theta}_{w}\right)$ can be obtained by projecting $f_{w}\left(\cdot \mid x^{N}\right)$ by KL divergence to the original exponential family. Hence, we refer to $\tilde{f}_{w}$ as the projected Bayes estimator. In information geometry [1] it is known that the -1-geodesic connecting $\tilde{f}_{w}\left(\cdot \mid x^{N}\right)$ and $f_{w}\left(\cdot \mid x^{N}\right)$ is orthogonal to the family $S$ and $\tilde{f}_{w}\left(\cdot \mid x^{N}\right)$ is referred to as the -1-projection of $f_{w}\left(\cdot \mid x^{N}\right)$ onto $S$.

In reference to the MDL estimator, in order to specify an estimator we have to specify a coding scheme for source distributions. Specifying a coding scheme is equivalent to specifying a discreet prior distribution, often obtained by discretizing a continuous distribution $w$ for the parameters. As discussed in Section V, the MDL estimates take the form $(*)$

$$
\hat{\theta}_{m d l}=\arg \min _{\theta}\left(-\ln p\left(x^{N} \mid \theta\right)-\ln (w(\theta) / \sqrt{I(\theta)})\right)
$$

where $I(\theta)$ is the determinant of Fisher information matrix (see also [15], [16], [2]). We refer to the estimator specified in such a way as the MDL estimator with the prior $w$. When $w$ is uniform over the coordinates, e.g., $\xi$, we say that the MDL estimator is taken with respect to the coordinate system $\xi$, since using that prior is equivalent to using the decimal expansion of $\xi$ 's value for coding the distribution. (A strict coding interpretation would require a proper prior that integrals (sums) to one over all $\theta$. Here we will use $(*$ ) also for improper priors such as the uniform over $\Re$.)

We have obtained the following result: Under a certain weak condition, "the MDL estimator with respect to the expectation parameter (as defined in Section II)" coincides with $\tilde{f}_{w_{J}}$ up to the $O(1 / N)$ term. This is the generalization of the equivalence between the two estimators for Bernoulli sources, because the parameter $r=p(1 \mid r)$ is the expectation parameter in that case. We also have shown that these estimators coincide with the bias-corrected maximum likelihood estimator (bias-corrected MLE, for short) [1] with respect to the canonical parameter (as defined in Section II) up to the $O(1 / N)$ term. These results not only supply an easy way to approximate the projected Bayes estimator, which is hard to calculate strictly, but characterize the maximin estimator on the basis of information geometry. That is, $\tilde{f}_{w_{J}}$ is nearly unbiased with respect to the canonical parameters. Moreover, we show that both the projected Bayes estimator with the uniform prior over the canonical parameter and the MDL estimator with Jeffreys prior equal the MLE ignoring terms of order $o(1 / N)$. Noting that the MLE is unbiased with respect to the expectation parameter, these results throw light on the symmetry between the projected Bayes estimator and the MDL estimator, because the natural and the expectation parameters of an exponential family form a dual pair from the point of view of information geometry [1]

\section{PRELIMINARIES}

The exponential family is defined as follows [5], [1].

Definition 1: Let $\nu$ be a $\sigma$-finite measure on the Borel subsets of $\Re^{n}$ and $\mathcal{X}$ be the support of $\nu$. Define

$$
\Theta_{a} \equiv\left\{\theta \mid \theta \in \Re^{n}, \int_{\mathcal{X}} \exp \left(\theta^{i} x_{i}\right) \nu(d x)<\infty\right\} .
$$

Let $\Theta$ denote a subset of $\Theta_{a}$. Define a function $\psi$ and a probability density $p$ on $\mathcal{X}$ with respect to $\nu$ by

$$
\psi(\theta) \equiv \ln \int_{\mathcal{X}} \exp \left(\theta^{i} x_{i}\right) \nu(d x)
$$

and

$$
p(x \mid \theta) \equiv \exp \left(\theta^{i} x_{i}-\psi(\theta)\right) .
$$

We refer to the set $S(\Theta) \equiv\{p(x \mid \theta) \mid \theta \in \Theta\}$ as an exponential family.

In the above definition and hereafter, we use Einstein's convention about summation, i.e., $\theta^{i} x_{i}$ denotes $\sum_{i=1}^{n} \theta^{i} x_{i}$ $\left(x_{i}\right.$ denotes the $i$ th component of $x$ ). Exponential families include many common statistical models such as Gauss distributions, Poisson distributions, Bernoulli sources, etc., where the role of $x$ is played by a suitable function of the original variables in these cases [5]. It is known that $\Theta_{a}$ is a convex set. Let $\mathcal{W}$ denote the closure of the convex hull of $\mathcal{X}$. It is known that we can assume that $\operatorname{dim} \mathcal{W}=\operatorname{dim} \Theta_{a}=n$ holds without loss of generality [5]. An exponential family which satisfies this condition is said to be minimal. We assume $S\left(\Theta_{a}\right)$ is minimal in this paper.

It is known that $\psi(\theta)$ is of class $C^{\infty}$ and strictly convex on $\Theta_{a}^{\circ}$ (where $A^{\circ}$ denotes the interior of $A \subseteq \Re^{n}$ ). We refer to $\theta$ as the canonical parameter (or $\theta$-coordinates). We define the expectation parameter (or $\eta$-coordinates) as $\eta_{i} \equiv E_{\theta}\left(x_{i}\right)$, where $E_{\theta}$ denotes the expectation with respect to $p(x \mid \theta)$. It is known that the function on $\Theta_{a}^{\circ}$ mapping $\theta \mapsto \eta$ is an injection and of class $C^{\infty}$. Let $H_{a}=\left\{\eta(\theta) \mid \theta \in \Theta_{a}^{\circ}\right\}$ be the range of this map. The parameters $\theta$ and $\eta$ have the 
geometrical interpretation as follows: $\theta$-coordinates are the affine coordinates for the 1-connection and $\eta$-coordinates is the affine coordinates for the -1-connection (see [1]).

We let $\partial_{i}$ and $\partial^{i}$, respectively, denote the differential operators $\partial / \partial \theta^{i}$ and $\partial / \partial \eta_{i}$. Note that $\partial_{i} \psi=\eta_{i}, \partial_{i} \partial_{j} \psi=g_{i j}$, and $\partial_{i} \partial_{j} \partial_{k} \psi=T_{i j k}$ hold on $\Theta_{a}^{\circ}$, where

$$
g_{i j}=E_{\theta}\left(\left(x_{i}-\eta_{i}\right)\left(x_{j}-\eta_{j}\right)\right)
$$

and

$$
T_{i j k}=E_{\theta}\left(\left(x_{i}-\eta_{i}\right)\left(x_{j}-\eta_{j}\right)\left(x_{k}-\eta_{k}\right)\right) .
$$

Further, $g_{i j}$ is the Fisher information matrix with respect to $\theta$. We let $g^{i j}$ denote the Fisher information matrix with respect to $\eta$. Then, $g_{i j} g^{j k}=\delta_{i}^{k}$ holds, i.e., $g^{i j}$ is the inverse matrix of $g_{i j}$. ( $\delta_{i}^{k}$ denotes the Kronecker's delta.) Finally, we note that the following holds:

$$
\partial_{i} \ln \left(\operatorname{det}\left|g_{i j}\right|\right)^{1 / 2}=T_{i j k} g^{j k} / 2 .
$$

In this paper, we refer to a function $f$ which maps

$$
\bigcup_{i=0,1, \cdots} \mathcal{X}^{i}
$$

to $\mathcal{H}$ (any set of probability distributions, referred to as a hypothesis set) as an estimator. Let $f$ be an estimator. We let $f\left[x^{N}\right]$ denote the image of $x^{N}$ by $f$ and $f\left[x^{N}\right](x)$ (or $f\left(x \mid x^{N}\right)$ ) denote the density of $f\left[x^{N}\right]$ at $x$. In particular, when $\mathcal{H} \subseteq S\left(\Theta_{a}\right), f$ is said to be proper.

We let $\hat{f}$ denote the MLE, i.e.,

$$
\hat{f}\left[x^{N}\right]=\arg \max _{p \in S\left(\Theta_{a}\right)} p\left(x^{N}\right) .
$$

We let $\hat{\eta}$ and $\hat{\theta}$, respectively, denote $\eta\left(\hat{f}\left[x^{N}\right]\right)$ and $\theta\left(\hat{f}\left[x^{N}\right]\right)$ for simplicity. Moreover, let $\hat{g}_{i n}, \hat{T}_{i j k}, \cdots$, respectively, denote their values at $\hat{f}\left[x^{N}\right]$. We let

$$
\bar{x}=\sum_{t=1}^{N} x_{t} / N
$$

where $x_{t}$ denotes the $t$ th observed value of $x$ (not the $t$ th component). This $\bar{x}$ is a sufficient statistic for $\Theta$. It is known that if $\bar{x} \in H_{a}$ holds, then the MLE $\hat{\eta}$ equals $\bar{x}$. Note that if an exponential family $S\left(\Theta_{a}\right)$ is "steep," then $H_{a}=\mathcal{W}^{\circ}$ holds. (If $E_{\theta}(|x|)=\infty$ holds for any $\theta \in \Theta_{a}-\Theta_{a}^{\circ}$, then $S\left(\Theta_{a}\right)$ is said to be steep, see for example [5].) When $S\left(\Theta_{a}\right)$ is steep, by the definition of $\mathcal{W}, \bar{x} \in \mathcal{W},=\bar{H}_{a}$ holds (where $\bar{A}$ denotes the closure of $A \subseteq \Re^{n}$ ). Strictly speaking, there does not exist $\hat{\eta}$ for $\bar{x} \in \partial H_{a}$, however, we define $\hat{\eta} \equiv \bar{x}$ for $\bar{x} \in \bar{H}_{a}$ for minimal steep exponential families (where $\partial A$ denotes the boundary of $A \subseteq \Re^{n}$ ).

Now we define prior distributions. Let $\Theta\left(\subseteq \Theta_{a}\right)$ be a connected open set of $\Re^{n}$ and $w(\theta) d \theta$ a prior distribution whose support is $\bar{\Theta}$. In the sequel, when we simply say "the prior $w$," it is supposed that $w$ is defined with respect to the measure $d \theta$. We assume that $w$ is of class $C^{2}$ on $\Theta$ and that $\int_{\Theta} w(\theta) d \theta$ does not necessarily equal 1 . Moreover, we permit the case that $\int_{\Theta} w(\theta) d \theta=\infty$ (improper prior). We define Jeffreys prior as $w_{J}(\theta) \equiv\left(\operatorname{det}\left|g_{i j}(\theta)\right|\right)^{1 / 2}$.

\section{THE BAYES EstimATOR}

We define the Bayes mixture estimator (Bayes predictor) with the prior $w$ as

$$
f_{w}\left[x^{N}\right](x) \equiv \int_{\Theta} p(x \mid \theta) w\left(\theta \mid x^{N}\right) d \theta
$$

where $w\left(\theta \mid x^{N}\right)$ is defined as

$$
p\left(x^{N} \mid \theta\right) w(\theta) / \int p\left(x^{N} \mid \theta\right) w(\theta) d \theta
$$

provided

$$
\int p\left(x^{N} \mid \theta\right) w(\theta) d \theta<\infty .
$$

We define the projection of $f_{w}$ to $S\left(\Theta_{a}\right)$ (denoted by $\tilde{f}_{w}$ ) as follows, where $D(p \| q)$ is defined as

$$
D(p \| q)=\int p(x) \ln (p(x) / q(x)) \nu(d x)
$$

(KL divergence):

$$
\tilde{f}_{w}\left[x^{N}\right] \equiv \arg \min _{p \in S\left(\Theta_{a}\right)} D\left(f_{w}\left[x^{N}\right] \| p\right) .
$$

We refer to $\tilde{f}_{w}$ as the projected Bayes estimator with the prior $w$. As we mentioned in the Introduction, $\tilde{f}_{w}$ equals the proper estimator $f$ minimizing $R_{N}(w, f)$.

The following Proposition holds.

Proposition 1: Suppose

$$
\int_{\Theta} p\left(x^{N} \mid \theta\right) w(\theta) d \theta<\infty
$$

Define

$$
\tilde{\eta} \equiv \int_{\Theta} \eta(\theta) w\left(\theta \mid x^{N}\right) d \theta .
$$

If $\tilde{\eta}$ is finite and belongs to $H_{a}$, then $\eta_{i}\left(\tilde{f}_{w}\left[x^{N}\right]\right)=\tilde{\eta}_{i}$ holds.

Proof: Noting $p(x \mid \theta)=\exp \left(\theta^{i} x_{i}-\psi(\theta)\right)$, we have

$$
\begin{aligned}
\int_{x \in \mathcal{X}} & f_{w}\left[x^{N}\right](x) \ln p(x \mid \theta) \nu(d x) \\
& =\int_{x \in \mathcal{X}} \int_{\xi \in \Theta} p(x \mid \xi) w\left(\xi \mid x^{N}\right) d \xi \cdot\left(\theta^{i} x_{i}-\psi(\theta)\right) \nu(d x) \\
& =\int_{\xi \in \Theta} \int_{x \in \mathcal{X}} p(x \mid \xi)\left(\theta^{i} x_{i}-\psi(\theta)\right) \nu(d x) \cdot w\left(\xi \mid x^{N}\right) d \xi \\
& =\int_{\xi \subset \Theta}\left(\theta^{i} \eta_{i}(\xi)-\psi(\theta)\right) w\left(\xi \mid x^{N}\right) d \xi \\
& =\theta^{i} \int_{\xi \in \Theta} \eta_{i}(\xi) w\left(\xi \mid x^{N}\right) d \xi-\psi(\theta) \\
& =\theta^{i} \cdot \tilde{\eta}_{i}-\psi(\theta) .
\end{aligned}
$$

Note that the estimate $\tilde{f}_{w}\left[x^{N}\right]$ maximizes

$$
\int_{\mathcal{X}} f_{w}\left[x^{N}\right](x) \ln p(x) \nu(d x)
$$

among $p$ in $S$. Since $\theta(\tilde{\eta})$ belongs to $\Theta_{a}$ by the assumption, $\theta$ which maximizes (2) is $\theta(\tilde{\eta})$, i.e., we obtain the proposition.

Q.E.D. 
Remark: If $S\left(\Theta_{a}\right)$ is steep, then $\tilde{\eta} \in H_{a}$ always holds whenever $\tilde{\eta}$ is finite.

We let $H_{\text {in }}$ denote a fixed compact set included in the interior of $H_{a}$ and $x^{\infty}$ an infinite sequence $x_{1} x_{2} \cdots$. Let $x^{N}$ denote the sequence which consists of the first $N$ elements of $x^{\infty}$. We define a class of $x^{\infty}, T_{N}$ as

$$
T_{N}=\left\{x^{\infty} \mid \forall I \geq N, \sum_{t=1}^{I} x_{t} / I \in H_{\text {in }}\right\} .
$$

We define a family of real-valued function $F_{\alpha}$ on $\mathcal{W} \times \Theta$ as

$$
F_{\alpha}(z, \theta)=\exp \left(\alpha\left(\theta^{i} z_{i}-\psi(\theta)\right)\right)
$$

where $\alpha \in \Re$. We can write $p\left(x^{N} \mid \theta\right)=F_{N}(\bar{x}, \theta)$.

We let $\Theta /\left\{\theta^{i}\right\}$ denote a set

$$
\left\{\left(\theta^{1}, \cdots, \theta^{i-1}, \theta^{i+1}, \cdots, \theta^{n}\right) \mid \theta \in \Theta\right\}
$$

and for

$$
\zeta(i)=\left(\zeta^{1}, \cdots, \zeta^{i-1}, \zeta^{i+1}, \cdots, \zeta^{n}\right) \in \Theta /\left\{\theta^{i}\right\}
$$

let $l_{\zeta(i)}$ denote a line in $\Re^{n}$ which is parallel to the $i$ th axis and goes through the point $\left(\zeta^{1}, \cdots, \zeta^{i-1}, 0, \zeta^{i+1}, \cdots, \zeta^{n}\right)$.

Now, we make the following assumptions:

Assumption 1: For any $i \in\{1, \cdots, n\}$ and almost all $\zeta(i) \in \Theta /\left\{\theta^{i}\right\}, l_{\zeta(i)} \cap \partial \Theta$ is a finite set.

Assumption 2: When $N$ is not less than a certain integer $N_{1}$, for any $z \in H_{\text {in }}, F_{N}(z, \theta) w(\theta)$ and $\eta(\theta) F_{N}(z, \theta) w(\theta)$ are integrable on $\Theta$.

Assumption 3: When $N$ is not less than a certain integer $N_{2}$, for any $z \in H_{i n},\left(\partial_{i} w(\theta)\right) F_{N}(z, \theta)$ is integrable on $\Theta$.

For example, if $\Theta$ is a convex set of $\Re^{n}$, then Assumption 1 holds. It also holds, if $\Theta$ can be decomposed to finite number of $n$-dimensional convex sets, i.e., $\bar{\Theta}=\bigcup_{1<i<r} \bar{\Theta}_{i}$ and $\Theta_{i} \cap \Theta_{j}=\emptyset(i \neq j)$ hold, where $r$ is a certain positive integer and each $\Theta_{i}$ is an $n$-dimensional open convex set.

Assumption 2 and 3 can be checked by using the following proposition.

Proposition 2: Let $\xi$ be a real-valued function on $\Theta$. For all sufficiently large $N, \forall z \in H_{\text {in }}$

$$
\int_{\Theta}\left|\xi(\theta) F_{N}(z, \theta)\right| d \theta<\infty
$$

holds, if and only if $\exists z \in H_{\text {in }}$

$$
\int_{\Theta}\left|\xi(\theta) F_{N}(z, \theta)\right| d \theta<\infty
$$

holds for a certain real $N$.

The proof is given in the Appendix.

Concerning the projected Bayes estimator, we obtain the following theorem.

Theorem 1: Suppose Assumptions 1-3 hold for an exponential family $S(\Theta)$ and a prior $w$. For any $N^{\prime} \in N I$

$$
\eta_{i}\left(\tilde{f}_{w}\left[x^{N}\right]\right)=\hat{\eta}_{i}+\partial_{i} \ln w(\hat{\theta}) / N+O(\sqrt{\ln N} / N \sqrt{N})
$$

holds unformly for $x^{\infty} \in T_{N^{\prime}}$. Especially when $w(\theta)$ is uniiform over $\Theta$

$$
\eta_{i}\left(\tilde{f}_{w}\left[x^{N}\right]\right)=\hat{\eta}_{i}+O\left(e^{-C N}\right)
$$

holds.
Remark: If $x^{\infty}$ is a sample of $p^{*} \in S\left(\Theta_{i n}^{\circ}\right)$, then we can show

$$
\lim _{N \rightarrow \infty} \operatorname{Pr}\left\{x^{\infty} \in T_{N}\right\}=1 .
$$

We give the proof in the next session.

We have the following corollary.

Corollary 1: Suppose Assumptions 1-3 hold for an exponential family $S(\Theta)$ and Jeffreys prior. For any $N^{\prime} \in N I$

$$
\eta_{i}\left(f_{w_{J}}\left[x^{N}\right]\right)=\hat{\eta}_{i}+\hat{T}_{i j k} \hat{g}^{j k} / 2 N+O(\sqrt{\ln N} / N \sqrt{N})
$$

holds, uniformly for $x^{\infty} \in T_{N^{\prime}}$.

Proof: Let $w(\theta)=w_{J}(\theta)$ in Theorem 1. Using (1), we obtain the claim of the corollary.

Q.E.D.

\section{PRoOf of TheOREM 1}

By differentiating

$$
F_{N}(\bar{x}, \theta) w(\theta)=\exp \left(N\left(\theta^{i} \bar{x}_{i}-\psi(\theta)\right)\right) w(\theta)
$$

with respect to $\theta^{k}$, we have

$$
\begin{aligned}
\partial_{k}(\exp & \left.\left(N\left(\theta^{i} \bar{x}_{i}-\psi(\theta)\right)\right) w(\theta)\right) \\
= & N\left(\bar{x}_{k}-\eta_{k}(\theta)\right) \exp \left(N\left(\theta^{i} \bar{x}_{i}-\psi(\theta)\right)\right) w(\theta) \\
& +\exp \left(N\left(\theta^{i} \bar{x}_{i}-\psi(\theta)\right)\right) \partial_{k} \ln w(\theta) \cdot w(\theta) \\
= & \left(N\left(\bar{x}_{k}-\eta_{k}(\theta)\right)+\partial_{k} \ln w(\theta)\right) F_{N}(\bar{x}, \theta) w(\theta) .
\end{aligned}
$$

Hence, we have

$$
\begin{aligned}
\partial_{k}\left(F_{N}(\bar{x}, \theta) w(\theta)\right)= & N \bar{x}_{k} F_{N}(\bar{x}, \theta) w(\theta) \\
& -N \eta_{k}(\theta) F_{N}(\bar{x}, \theta) w(\theta) \\
& +\partial_{k} \ln w(\theta) \cdot F_{N}(\bar{x}, \theta) w(\theta) .
\end{aligned}
$$

Since the fact that $\bar{x} \in H_{\text {in }}$ holds for $N \geq N^{\prime}$ and Assumptions 2 and 3, we can see that the right-hand side is integrable for sufficiently large $N$. Therefore, the left handside is also integrable. Hence, integrating both sides over $\Theta$, we have

$$
\begin{aligned}
\int \partial_{k}\left(F_{N}(\bar{x}, \theta) w(\theta)\right) d \theta= & N \bar{x}_{k} \int F_{N}(\bar{x}, \theta) w(\theta) d \theta \\
& -N \int \eta_{k}(\theta) F_{N}(\bar{x}, \theta) w(\theta) d \theta \\
& +\int \partial_{k} \ln w(\theta) \cdot F_{N}(\bar{x}, \theta) w(\theta) d \theta .
\end{aligned}
$$

Dividing both sides by $\int_{\Theta} F_{N}(\bar{x}, \theta) w(\theta) d \theta$ and letting

$$
w_{N}(\theta \mid z) \equiv F_{N}(z, \theta) w(\theta) / \int F_{N}(z, \theta) w(\theta) d \theta
$$

$\left(w_{N}(\theta \mid \bar{x})\right.$ equals the posterior density), we have

$\int_{\Theta} \partial_{k} w_{N}(\theta \mid \bar{x}) d \theta=N \bar{x}_{k}-N \tilde{\eta}_{k}+\int_{\Theta} \partial_{k} \ln w(\theta) \cdot w_{N}(\theta \mid \bar{x}) d \theta$.

Namely, we obtain

$$
\begin{aligned}
\tilde{\eta}_{k}= & \bar{x}_{k}+\int \partial_{k} \ln w(\theta) \cdot w_{N}(\theta \mid \bar{x}) d \theta / N \\
& -\int \partial_{k} w_{N}(\theta \mid \bar{x}) d \theta / N
\end{aligned}
$$


We can prove the following two formulas:

$$
\int_{\Theta} \partial_{k} \ln w(\theta) \cdot w_{N}(\theta \mid z) d \theta=\partial_{k} \ln w\left(\theta_{z}\right)+O(\sqrt{\ln N} / \sqrt{N})
$$

and

$$
\int_{\Theta} \partial_{k} w_{N}(\theta \mid z) d \theta=O(\exp (-D N))
$$

where we let $\theta_{z}$ denote $\left.\theta\right|_{\eta=z}$ and $D$ is a certain constant. These hold uniformly for $z \in H_{i n}$. By the assumption, $\bar{x}$ in (3) belongs to $H_{\text {in }}$ for $N \geq N^{\prime}$. Therefore, the right-hand side of (3) belongs to $H_{a}$ for sufficiently large $N$ under (4) and (5), i.e., we can obtain the claim of the theorem.

Now, we show (4). Hereafter in this section, we let $\hat{\theta}$ denote $\theta_{z}=\left.\theta\right|_{\eta=z}$. Let $G_{N}$ denote the left-hand side of (4) and let $h(\theta) \equiv \partial_{k} \ln w(\theta)$, then we have

$$
G_{N}=\int h(\theta) F_{N}(z, \theta) w(\theta) d \theta / \int F_{N}(z, \theta) w(\theta) d \theta .
$$

Let $\delta=((n+1) \ln N /(2 N))^{1 / 2}$ and define

$$
M_{\delta} \equiv\left\{\theta \mid \hat{g}_{i j}\left(\theta^{i}-\hat{\theta}^{i}\right)\left(\theta^{j}-\hat{\theta}^{j}\right) \leq \delta^{2}\right\} .
$$

( $\hat{g}_{i j}$ denotes its value at $\left.\eta=z.\right)$ By Taylor's theorem, we have

$$
\begin{aligned}
\ln F_{N}(z, \theta)= & \ln F_{N}(z, \hat{\theta})-(N / 2) \hat{g}_{i j}\left(\theta^{i}-\hat{\theta}^{i}\right)\left(\theta^{j}-\hat{\theta^{j}}\right) \\
& +N O\left(|\theta-\hat{\theta}|^{3}\right) .
\end{aligned}
$$

Since $\Theta_{i n}$ is compact and $g_{i j}$ is of class $C^{\infty}$ on $\Theta_{a}^{\circ}$, $\left|\partial_{l} g_{i j}(\theta)\right|<\infty$ holds uniformly on $\Theta_{i n}$. Then, the following holds for an arbitrary $\theta$ in $M_{\delta}$ :

$$
\begin{aligned}
F_{N}(z, \theta) & =F_{N}(z, \hat{\theta}) e^{-(N / 2) \hat{g}_{i j}\left(\theta^{i}-\hat{\theta^{i}}\right)\left(\theta^{j}-\hat{\theta^{j}}\right)+O\left(N \delta^{3}\right)} \\
& =\left(1+O\left(N \delta^{3}\right)\right) F_{N}(z, \hat{\theta}) e^{-(N / 2) \hat{g}_{i j}\left(\theta^{i}-\hat{\theta}^{i}\right)\left(\theta^{j}-\hat{\theta^{j}}\right)}
\end{aligned}
$$

Here, though the term $O\left(\delta^{3}\right)$ depends also on $\theta$, the order of $\delta$ is uniform with respect to $\theta$. Hence, hereafter, we use the order notation for $\delta$ in the same sense.

We also have $\forall \theta \in \partial M_{\delta}$

$$
\begin{aligned}
\ln F_{N}\left(z, \hat{\theta}-\ln F_{N}(z, \theta) \geq\right. & (N / 2) \hat{g}_{i j}\left(\theta^{i}-\hat{\theta^{i}}\right)\left(\theta^{j}-\hat{\theta^{j}}\right) \\
& +N \cdot O\left(|\theta-\hat{\theta}|^{3}\right) \\
= & N \delta^{2} / 2+O\left(N \delta^{3}\right) .
\end{aligned}
$$

Hence, by the fact that $\psi(\theta)-\theta^{i} \bar{x}_{i}$ is strictly convex on a convex set $\Theta_{a}^{\circ}$, the following holds for an arbitrary $\theta$ in $\Theta-M_{\delta}$ :

$$
F_{N}(z, \theta) \leq\left(1+O\left(N \delta^{3}\right)\right) F_{N}(z, \hat{\theta}) \exp \left(-N \delta^{2} / 2\right) .
$$

Now, we evaluate the numerator of $G_{N}$. We have

$$
\begin{aligned}
\int_{\Theta} h(\theta) F_{N}(z, \theta) w(\theta) d \theta= & \int_{M_{\delta}} h(\theta) F_{N}(z, \theta) w(\theta) d \theta \\
& +\int_{\Theta-M_{\delta}} h(\theta) F_{N}(z, \theta) w(\theta) d \theta
\end{aligned}
$$

By Taylor's theorem, $\forall \theta \in M_{\delta}$

$$
\ln w(\theta)=\ln w(\hat{\theta})+\left(\theta^{i}-\hat{\theta}^{i}\right) \partial_{i} w\left(\theta^{\prime}\right) / w\left(\theta^{\prime}\right)
$$

holds, where $\theta^{\prime}=\alpha \theta+(1-\alpha) \hat{\theta}$ for a certain $\alpha \in[0,1]$. Since $w$ is of class $C^{2}$ and $w>0$ on $\Theta$ and $\Theta_{i n}$ is compact, we have $\left|\partial_{i} w(\theta) / w(\theta)\right|<\infty$ on $\Theta_{i n}$. Therefore, $\forall \theta \in M_{\delta}$

$$
\ln w(\theta)=\ln w(\hat{\theta})+O(\delta)
$$

i.e., $\forall \theta \in M_{\delta}$

$$
w(\theta)=(1+O(\delta)) w(\hat{\theta})
$$

holds. Similarly, we also obtain $\forall \theta \in M_{\delta}$

$$
h(\theta)=h(\hat{\theta})+O(\delta) .
$$

Then, using (6), we can transform the first term of the righthand side of (8) as follows:

$$
\begin{aligned}
\int_{M_{\delta}} h(\theta) F_{N}(z, \theta) w(\theta) d \theta= & A B C \cdot F_{N}(z, \hat{\theta}) w(\hat{\theta}) \\
& \cdot \int_{M_{\delta}} e^{-(N / 2) \hat{g}_{i j}\left(\theta^{i}-\hat{\theta}^{i}\right)\left(\theta^{j}-\hat{\theta}^{j}\right)} d \theta
\end{aligned}
$$

where we let

$$
\begin{aligned}
& A=h(\hat{\theta})+O(\delta) \\
& B=1+O(\delta)
\end{aligned}
$$

and

$$
C=1+O\left(N \delta^{3}\right)
$$

Moreover, we have

$$
\begin{aligned}
& \int_{M_{\delta}} e^{-(N / 2) \hat{g}_{i j}\left(\theta^{i}-\hat{\theta}^{i}\right)\left(\theta^{j}-\hat{\theta}^{j}\right)} d \theta \\
&=\int_{\Re^{n^{n}}} e^{-(N / 2) \hat{g}_{i j}\left(\theta^{i}-\hat{\theta}^{i}\right)\left(\theta^{j}-\hat{\theta^{j}}\right)} d \theta \\
&-\int_{M_{\delta}^{c}} e^{-(N / 2) \hat{g}_{i j}\left(\theta^{i}-\hat{\theta}^{i}\right)\left(\theta^{j}-\hat{\theta^{j}}\right)} d \theta \\
&=(2 \pi / \hat{g} N)^{n / 2}-\int_{M_{\delta}^{c}} e^{-(N / 2) \hat{g}_{i j}\left(\theta^{i}-\hat{\theta}^{i}\right)\left(\theta^{j}-\hat{\theta}^{j}\right)} d \theta
\end{aligned}
$$

where we let $\hat{g}$ denote $\operatorname{det}\left|\hat{g}_{i j}\right|$ and $M_{\delta}^{c}$ denote $\Re^{n}-M_{\delta}$. Here, we have

$$
\begin{aligned}
& \int_{M_{\delta}^{c}} e^{-(N / 2) \hat{g}_{i j}\left(\theta^{i}-\hat{\theta}^{i}\right)\left(\theta^{j}-\hat{\theta^{j}}\right)} d \theta \\
& \leq e^{-(N-1) \delta^{2} / 2} \int_{M_{\delta}^{c}} e^{-(1 / 2) \hat{g}_{i j}\left(\theta^{i}-\hat{\theta}^{i}\right)\left(\theta^{j}-\hat{\theta^{j}}\right)} d \theta=O\left(e^{-N \delta^{2}}\right) .
\end{aligned}
$$

Therefore, we have

$$
\int_{M_{\delta}} e^{-(N / 2) \hat{g}_{i j}\left(\theta^{i}-\hat{\theta}^{i}\right)\left(\theta^{j}-\hat{\theta^{j}}\right)} d \theta=(2 \pi / \hat{g} N)^{n / 2}-O\left(e^{-N \delta^{2}}\right) .
$$

Hence, noting $A B C=O(1)$, we obtain

$$
\begin{aligned}
& \int_{M_{\delta}} h(\theta) F_{N}(z, \theta) w(\theta) d \theta \\
& \quad=F_{N}(z, \hat{\theta}) w(\hat{\theta})\left(A B C(2 \pi / \hat{g} N)^{n / 2}-O\left(e^{-N \delta^{2}}\right)\right) .
\end{aligned}
$$


Noting (7) and

$$
\int_{\Theta}\left|h(\theta) F_{N}(z, \theta) w(\theta)\right| d \theta<\infty \quad \text { for } N \geq \max \left\{N_{2}, N_{3}\right\}
$$

we have

$$
\begin{aligned}
\left|\int_{\Theta-M_{\delta}} h(\theta) F_{N}(z, \theta) w(\theta) d \theta\right| & \leq \int_{\Theta-M_{\delta}}\left|h(\theta) F_{N}(z, \theta) w(\theta)\right| d \theta \\
& =F_{N}(z, \hat{\theta}) O\left(e^{-N \delta^{2}}\right) \\
& =F_{N}(z, \hat{\theta}) w(\hat{\theta}) O\left(e^{-N \delta^{2}}\right) .
\end{aligned}
$$

(The last equality follows from the fact that $1 / w(\theta)<\infty$ on $\left.\Theta_{i n}.\right)$ Now, we obtain

$$
\begin{aligned}
& \int_{\Theta} h(\theta) F_{N}(z, \theta) w(\theta) d \theta \\
& \quad=F_{N}(z, \hat{\theta}) w(\hat{\theta})\left(A B C(2 \pi / \hat{g} N)^{n / 2}+O\left(e^{-N \delta^{2}}\right)\right) .
\end{aligned}
$$

Next, we consider the denominator of $G_{N}$. We can similarly evaluate that by plugging in 1 to $A$ except for the evaluation of

$$
\int_{\Theta-M_{\delta}} F_{N}(z, \theta) w(\theta) d \theta
$$

Now, noting (7) and

$$
\int_{\Theta}\left|F_{N}(z, \theta) w(\theta)\right| d \theta<\infty, \quad \text { for } N \geq N_{1}
$$

we have

$$
\begin{aligned}
\int_{\Theta-M_{\delta}} F_{N}(z, \theta) w(\theta) d \theta & =F_{N}(z, \hat{\theta}) O\left(e^{-N \delta^{2}}\right) \\
& =F_{N}(z, \hat{\theta}) w(\hat{\theta}) O\left(e^{-N \delta^{2}}\right)
\end{aligned}
$$

Namely, we obtain

$$
\begin{aligned}
& \int_{\Theta} F_{N}(z, \theta) w(\theta) d \theta \\
& \quad=F_{N}(z, \hat{\theta}) w(\hat{\theta})\left(B^{\prime} C^{\prime}(2 \pi / \hat{g} N)^{n / 2}+O\left(e^{-N \delta^{2}}\right)\right)
\end{aligned}
$$

where $B^{\prime}=1+O(\delta)$ and $C^{\prime}=1+O\left(N \delta^{3}\right)$.

Now, using (11) and (10), we obtain

$$
\begin{aligned}
G_{N} & =\frac{A B C(2 \pi / \hat{g} N)^{n / 2}+O\left(e^{-N \delta^{2}}\right)}{B^{\prime} C^{\prime}(2 \pi / \hat{g} N)^{n / 2}+O\left(e^{-N \delta^{2}}\right)} \\
& =\frac{A B C+O\left(N^{n / 2} e^{-N \delta^{2}}\right)}{B^{\prime} C^{\prime}+O\left(N^{n / 2} e^{-N \delta^{2}}\right)}
\end{aligned}
$$

Hence, noting $\delta^{2}=(n+1) \ln N /(2 N)$, we have

$$
\begin{aligned}
& N^{n / 2} e^{-N \delta^{2}}=O(1 / \sqrt{N}) \\
& \delta=O\left((\ln N / N)^{1 / 2}\right)
\end{aligned}
$$

and

$$
N \delta^{3}=O\left((\ln N / N)^{1 / 2}\right)
$$

Then, we have

$$
B^{\prime} C^{\prime}=1+O\left((\ln N / N)^{1 / 2}\right)
$$

and

$$
A B C=h(\hat{\theta})+O\left((\ln N / N)^{1 / 2}\right) .
$$

Therefore, we have

$$
\begin{aligned}
G_{N} & =\left(h(\hat{\theta})+O\left((\ln N / N)^{1 / 2}\right)\right) /\left(1+O\left((\ln N / N)^{1 / 2}\right)\right) \\
& =h(\hat{\theta})+O\left((\ln N / N)^{1 / 2}\right) .
\end{aligned}
$$

Namely, we have (4).

Next, we show (5). Suppose $k=1$ for simplicity. Let

$$
I \equiv \int_{\Theta}\left(\partial_{1} w_{N}(\theta \mid z)\right) d \theta
$$

By Assumption 1, for almost all $\zeta(1) \in \Theta /\left\{\theta^{1}\right\}, l_{\zeta(1)} \cap \partial \Theta$ is a finite set. Let $Q(\zeta(1))$ denote $l_{\zeta(1)} \cap \partial \Theta$. We let $Z$ denote the largest subset of $\Theta /\left\{\theta^{1}\right\}$, such that $Q(\zeta(1))$ is a finite set for all $\zeta(1) \in Z$. Then, integrating $\partial_{1} w_{N}(\theta \mid z)$ with respect to $\theta^{1}$ (Fubini's theorem), we obtain

$$
I=\int_{\zeta \in Z} \sum_{\mu \in Q(\zeta)} \epsilon(\mu)\left(w_{N}(\mu \mid z)\right) d \zeta
$$

where $\epsilon(\mu)$ is +1 or -1 . Hence, we have

$$
\begin{aligned}
|I| & \leq \int_{\zeta \in Z} \sum_{\mu \in Q(\zeta)} w_{N}(\mu \mid z) d \zeta \\
& =\frac{\int_{\zeta \in Z} \sum_{\mu \in Q(\zeta)} F_{N}(z, \mu) w(\mu) d \zeta}{\int_{\theta \in \Theta} F_{N}(z, \theta) w(\theta) d \theta} .
\end{aligned}
$$

Since $x^{\infty} \in T_{N^{\prime}}, \hat{\theta}=\left.\theta\right|_{\eta=z} \in \Theta_{\text {in }} \subset \Theta^{\circ}$ holds for all $N \geq N^{\prime}$. Hence, $\partial \Theta \cap M_{\delta}=\emptyset$ holds for large $N$. Hence from (7), we have $\forall \theta \in \partial \Theta$

$$
F_{N}(z, \theta) \leq\left(1+O\left(N \delta^{3}\right)\right) F_{N}(z, \hat{\theta}) \exp \left(-N \delta^{2} / 2\right)
$$

Therefore, noting $\mu \in Q(\zeta) \subset \partial \Theta$ and

$$
\int_{\zeta \in Z} \sum_{\mu \in Q(\zeta)} F_{N}(z, \mu) w(\mu) d \zeta<\infty
$$

for $N>\max \left\{N_{1}, N_{2}\right\}$, we have

$$
\int_{\zeta \in Z} \sum_{\mu \in Q(\zeta)} F_{N}(z, \mu) w(\mu) d \zeta=F_{N}(z, \hat{\theta}) O\left(\exp \left(-D^{\prime} N\right)\right)
$$

( $D^{\prime}$ is a certain constant.) Together with (12) and (11), we can write

$$
|I|=O\left(N^{n / 2} \exp \left(-D^{\prime} N\right)\right)=O(\exp (-D N))
$$

where $D$ is a certain constant. This concludes (5). Now we have obtained the claim of the theorem.

Q.E.D. 


\section{THE MDL ESTIMATOR}

In this section, we construct an estimator for the $n$ dimensional model $S=\{p(x \mid \theta) \mid \theta \in \Theta\}$ (it is not necessarily an exponential family) based on the MDL principle, i.e., we determine the code (quantization) for parameters based on the MDL principle. The argument in this section is essentially parallel to those of Rissanen [12], Barron [2], and Wallace and Freeman [16]. The point that is unique to our derivation is that we determine the code so as to minimize the average total code length. In [12] and [2], the typical value of total code length was minimized. In [16], the expectation (by the prior distribution) of the average total code length was minimized.

Suppose the prior $d \phi=w(\theta) d \theta$ over $\Theta$. The coding of the parameter $\theta$ consists of two parts: 1) quantizing $\Theta$ (to obtain a countable set) and 2) describing quantized points. Since the optimal coding depends on the data size $N$, we let the quantization depend on $N$ and let $\Theta_{N}$ denote the set of quantized points. (We suppose that $N$ is known prior to encoding.) Let $r(\bar{\theta})$ denote the region represented by the quantized point $\bar{\theta}$ and let $v(\bar{\theta})=\int_{\theta \in r(\bar{\theta})} w(\theta) d \theta$. Moreover, we give the code length $l_{N}(\bar{\theta})=-\ln v(\bar{\theta})$ to the quantized point $\bar{\theta}$. We let $\mathcal{L}_{N}$ denote the set of such coding schema, obtained by varying the quantizations $\Theta_{N}$. Now, we give the definition of MDL estimator.

Definition 2: Define $\theta_{m d l}$ as follows:

$$
\begin{aligned}
\hat{\theta}_{l_{N}} & \equiv \arg \min _{\bar{\theta} \subset \Theta_{N}}\left(-\ln p\left(x^{N} \mid \bar{\theta}\right)+l_{N}(\bar{\theta})\right), \\
l_{N}^{*} & \equiv \arg \min _{l_{N} \in \mathcal{L}_{N}} E_{\theta}\left(-\ln p\left(x^{N} \mid \hat{\theta}_{l_{N}}\right)+l_{N}\left(\hat{\theta}_{l_{N}}\right)\right), \\
\theta_{m d l}\left(x^{N}\right) & \equiv \arg \min _{\bar{\theta}}\left(-\ln p\left(x^{N} \mid \bar{\theta}\right)+l_{N}^{*}(\bar{\theta})\right) .
\end{aligned}
$$

We refer to the function which maps $x^{N} \in \mathcal{X}^{N}$ to $f_{m d l}^{w}\left[x^{N}\right] \equiv$ $p\left(\cdot \mid \theta_{m d l}\left(x^{N}\right)\right)$ as the MDL estimator with the prior $w$. Especially, when $w$ is uniform over $\Theta$, we refer to $f_{m d l}^{w d \theta}$ as the MDL estimator with respect to the coordinate system $\theta$ and let $f_{m d l}^{\theta}$ denote it.

Remark: In the following approximation, the code length $l^{*}$ does not depend on $\theta$.

Let us determine on approximation to $l_{N}^{*}$. To this end we shall obtain the conditional expected value of total description length $\mathrm{DL}(\bar{\theta})$ under the condition $\hat{\theta}_{l_{N}} \in r(\bar{\theta})$. The code length for the parameter is given by $-\ln v(\bar{\theta})$ and the code length for the data $x^{N}$ is given by $-\ln p\left(x^{N} \mid \hat{\theta}_{l_{N}}\right)$. Let $g_{i j}$ denote the Fisher information matrix with respect to $\theta$. We suppose that $r(\bar{\theta})$ 's can be approximated by rectangles each axis of which lies in the direction of principal axis of $g_{i j}(\bar{\theta})$. (If not the case, the description length becomes longer. See [16].) Let $X_{\alpha}$ denote the unit tangent vector along the $\alpha$ th principal axis of $g_{i j}(\theta)$, and $\lambda_{\alpha}$ denote the eigenvalue associated with the $\alpha$ th principal axis. Let $d_{\alpha}$ be a smooth function defined on $\Theta$ and suppose that $d_{\alpha}(\bar{\theta})$ equals the length of the $X_{\alpha}$ direction's axis of $r(\bar{\theta})$. Then

$$
v(\bar{\theta}) \sim \prod_{\alpha=1}^{n} d_{\alpha}(\bar{\theta}) w(\bar{\theta})
$$

holds. (Hereafter, we define

$$
v(\hat{\theta}) \equiv \prod_{\alpha=1}^{n} d_{\alpha}(\hat{\theta}) w(\hat{\theta})
$$

for $\hat{\theta} \notin \Theta_{N}$.) By Taylor expansion, we have

$$
\ln p\left(x^{N} \mid \hat{\theta}_{l_{N}}\right) \sim \ln p\left(x^{N} \mid \hat{\theta}\right)-(N / 2) g_{i j}\left(\hat{\theta}_{l_{N}}^{i}-\hat{\theta}^{i}\right)\left(\hat{\theta}_{l_{N}}^{j}-\hat{\theta}^{j}\right) .
$$

We suppose that the conditional distribution of $\hat{\theta}$ given $\hat{\theta} \in$ $r(\bar{\theta})$ is approximately uniform so that

$$
E_{\theta}\left[g_{i j}\left(\hat{\theta}_{l_{N}}^{i}-\hat{\theta}^{i}\right)\left(\hat{\theta}_{l_{N}}^{j}-\hat{\theta}^{j}\right) \mid \hat{\theta}_{l_{N}}=\bar{\theta}\right]
$$

almost equals

$$
\sum_{\alpha} \lambda_{\alpha}(\bar{\theta}) d_{\alpha}(\bar{\theta})^{2} / 12
$$

Then, we obtain

$$
\begin{aligned}
\operatorname{DL}(\bar{\theta}) \sim & E_{\theta}\left[-\ln p\left(x^{N} \mid \hat{\theta}\right) \mid \hat{\theta}_{l_{N}}=\bar{\theta}\right] \\
& +(N / 24) \sum_{\alpha} \lambda_{\alpha}(\bar{\theta}) d_{\alpha}(\bar{\theta})^{2}-\ln v(\bar{\theta}) .
\end{aligned}
$$

Hence, we can evaluate the (unconditional) expectation of the total description length (denoted by $L$ ) as follows:

$$
\begin{aligned}
L= & \sum_{\bar{\theta}} \operatorname{DL}(\bar{\theta}) \operatorname{Pr}\left\{\hat{\theta}_{l_{N}}=\bar{\theta}\right\} \\
\sim & E_{\theta}\left(-\ln p\left(x^{N} \mid \hat{\theta}\right)\right)+\sum_{\bar{\theta}}(-\ln v(\bar{\theta}) \\
& \left.+\sum_{\alpha} N \lambda_{\alpha}(\bar{\theta}) d_{\alpha}(\bar{\theta})^{2} / 24\right) \operatorname{Pr}\left\{\hat{\theta}_{l_{N}}=\bar{\theta}\right\} .
\end{aligned}
$$

Since

$$
\begin{aligned}
-\ln v(\bar{\theta}) & +\sum_{\alpha} N \lambda_{\alpha}(\bar{\theta}) d_{\alpha}(\bar{\theta})^{2} / 24 \\
& \sim E_{\theta}\left[-\ln v(\hat{\theta})+\sum_{\alpha} N \lambda_{\alpha}(\hat{\theta}) d_{\alpha}(\hat{\theta})^{2} / 24 \mid \hat{\theta} \in r(\bar{\theta})\right]
\end{aligned}
$$

and

$$
\operatorname{Pr}\left\{\hat{\theta}_{l_{N}}=\bar{\theta}\right\} \sim \operatorname{Pr}\{\hat{\theta} \in r(\bar{\theta})\}
$$

we obtain

$$
\begin{aligned}
\sum_{\bar{\theta}}\left(\sum_{\alpha} N \lambda_{\alpha}(\bar{\theta}) d_{\alpha}(\bar{\theta})^{2} / 24-\ln v(\bar{\theta})\right) \operatorname{Pr}\left\{\hat{\theta}_{l_{N}}=\bar{\theta}\right\} \\
\sim E_{\theta}\left[\sum_{\alpha}\left(N \lambda_{\alpha}(\hat{\theta}) d_{\alpha}(\hat{\theta})^{2} / 24-\ln d_{\alpha}(\hat{\theta})\right)\right]
\end{aligned}
$$

i.e., we have

$$
L \sim E_{\theta}\left[\sum_{\alpha}\left(N \lambda_{\alpha}(\hat{\theta}) d_{\alpha}(\hat{\theta})^{2} / 24-\ln d_{\alpha}(\hat{\theta})\right)\right]+C_{\theta}
$$

where we let $C_{\theta}=E_{\theta}\left(-\ln p\left(x^{N} \mid \hat{\theta}\right)-\ln w(\hat{\theta})\right)$. The choice of $d_{\alpha}(\hat{\theta})$ minimizes this approximated code length is $d_{\alpha}=\left(12 /\left(N \lambda_{\alpha}\right)\right)^{1 / 2}$. Hence, we have

$$
v(\theta)=\prod_{\alpha=1}^{n} d_{\alpha}(\theta) w(\theta)=12^{n / 2} N^{-n / 2}\left(\operatorname{det}\left|g_{i j}\right|\right)^{-1 / 2} .
$$


Finally, we obtain

$$
\begin{aligned}
l^{*}(\bar{\theta}) \sim & (1 / 2) \cdot \ln \operatorname{det}\left|g_{i j}(\bar{\theta})\right| \\
& +(n / 2) \ln N-\ln w(\bar{\theta})-(n \ln 12) / 2 .
\end{aligned}
$$

Then, we have

$$
\begin{aligned}
& \theta_{m d l}=\arg \min _{\bar{\theta} \in \Theta_{N}}(-\ln p\left(x^{N} \mid \bar{\theta}\right)+(1 / 2) \\
&\left.\cdot \ln \operatorname{det}\left|g_{i j}(\bar{\theta})\right|-\ln w(\bar{\theta})\right) .
\end{aligned}
$$

The above length $l^{*}(\bar{\theta})$ is essentially equivalent to the ones given in [15], [16], [2] (where a term of order $O(\ln \ln N)$ is included, because of the lack of the assumption that $N$ is known).

In the sequel, we neglect the quantization error. Namely, we employ the following as the definition of the MDL estimator:

$$
\hat{\theta}_{m d l} \equiv \arg \min _{\theta \in \Theta}\left(-\ln p\left(x^{N} \mid \theta\right)+\frac{\ln \operatorname{det}\left|g_{i j}(\theta)\right|}{2}-\ln w(\theta)\right) .
$$

We can rewrite the above definition as

$$
\hat{\theta}_{m d l}=\arg \max _{\theta \in \Theta} p\left(x^{N} \mid \theta\right) w(\theta) /\left(\operatorname{det}\left|g_{i j}(\theta)\right|\right)^{1 / 2} .
$$

Hence, the estimator $\hat{\theta}_{m d l}$ is equivalent to the posterior mode estimator provided that posterior density is defined with respect to the measure $\left(\operatorname{det}\left|g_{i j}(\theta)\right|\right)^{1 / 2} d \theta$, which is the natural volume element of $S$. Therefore, $f_{m d l}^{w}$ is invariant under the transformation of coordinate system.

Let $\rho(\theta)$ denote $w(\theta) /\left(\operatorname{det}\left|g_{i j}\right|\right)^{1 / 2}$ and define a family of real-valued function $L_{\alpha}(z, \theta)$ on $\mathcal{W} \times \Theta$ as

$$
L_{\alpha}(z, \theta)=-\alpha\left(\theta^{i} z_{i}-\psi(\theta)\right)-\ln \rho(\theta)
$$

where $\alpha \in \Re$. Then, $L_{N}(\bar{x}, \theta)$ equals the total description length for the MDL estimator with the prior $w$. We assume the following.

Assumption 4: When $N$ is not less than a certain integer $N_{3}$, for any $z \in H_{\text {in }}$

$$
\arg \min _{\theta \in \Theta} L_{N}(z, \theta) \in \Theta^{\circ}
$$

holds.

This assumption can be checked by using the following proposition.

Proposition 3: Assumption 4 holds if and only if the following holds: For a certain $N_{4} \in N I$, for a certain $z \in H_{\text {in }}$

$$
\arg \min _{\theta \in \Theta} L_{N}(z, \theta) \in \Theta^{\circ}
$$

holds.

The proof is similar to that of Proposition 2, we omit it.

We can prove the following lemma.

Lemma 1: Suppose Assumptions 1 and 4 hold for $S(\Theta)$ and $w$. For any $N^{\prime} \in N I$

$\eta_{i}\left(f_{m d l}^{w}\left[x^{N}\right]\right)=\hat{\eta}_{i}+\left(\partial_{i} \ln w(\hat{\theta})-\hat{T}_{i j k} \hat{g}^{j k} / 2\right) / N+O\left(1 / N^{2}\right)$

holds, uniformly for $x^{\infty} \in T_{N^{\prime}}$.
Proof: Let us prove that arg $\min _{\eta \in H} L_{N}(z, \theta(\eta))$ equals $z_{i}+\left.\partial_{i} \ln \rho(\theta)\right|_{\eta=z} / N+O\left(1 / N^{2}\right)$ uniformly on $H_{i n}$. That implies the claim of the Lemma.

Let $\theta_{z}$ denote $\left.\theta\right|_{\eta=z}$. Define $K_{\epsilon}\left(\theta_{z}\right)=\left\{\theta|| \theta-\theta_{z} \mid<\epsilon\right\}$. First, we show that for any small $\epsilon$, there exists $N_{a}$ such that $\forall N>N_{a}$, arg $\min _{\theta \in \Theta} L_{N}(z, \theta) \in K_{\epsilon}\left(\theta_{z}\right)$. Since $w(\theta)$ is of class $C^{2}, B \equiv \sup _{\theta \in \Theta_{i n}}(-\ln \rho(\theta))<\infty$ holds. Let $l_{z}=\theta_{z}^{i} z_{i}-\psi\left(\theta_{z}\right)$, then we have $L_{N}\left(z, \theta_{z}\right) \leq N l_{z}+B$. Since Assumption 4, we have $\forall \theta \in \Theta_{a}, L_{N}(z, \theta) \geq A$ for $N=N_{3}$, where $A$ is a certain real. Then, we have

$$
-\ln \rho(\theta) \geq A+N_{3}\left(\theta^{i} \bar{x}_{i}-\psi(\theta)\right)
$$

Hence, $L_{N}(z, \theta) \geq-\left(\theta^{i} z_{i}-\psi(\theta)\right)\left(N-N_{3}\right)+A$ holds. Note that $-\left(\theta^{i} z_{i}-\psi(\bar{\theta})\right)$ is strictly convex on $\Theta_{a}^{\circ}$ and that $\Theta_{a}^{\circ}$ is a convex set. For any small $\epsilon$, there exists $\delta>0$ such that $\forall \theta \in\left(\Theta_{a}-M_{\epsilon}\right),-\left(\theta^{i} \bar{x}_{i}-\psi(\theta)\right) \geq-\hat{l}+\delta$ holds. Then, we have $\forall N>N_{3}, \forall \theta \in \Theta_{a}-M_{\epsilon}$

$$
\begin{aligned}
L_{N}(z, \theta) & \geq\left(-l_{z}+\delta\right)\left(N-N_{3}\right)+A \\
& =-N l_{z}+A-N_{3}(-\hat{l}+\delta)+N \delta .
\end{aligned}
$$

Hence, for sufficiently large $N, \forall \theta \in\left(\Theta_{a}-M_{\epsilon}\right), L_{N}(z, \theta) \geq$ $L_{N}\left(z, \theta_{z}\right)$ holds. Therefore, $\arg \min _{\theta \in \Theta} L_{N}(z, \theta) \in K_{\epsilon}\left(\theta_{z}\right)$ holds. Since $H_{i n}$ is included in $H^{\circ}$, the above argument uniformly holds for $z \in H_{i n}$.

Let $\epsilon_{0}$ be small such that $K_{\epsilon_{0}}(\theta) \subset \Theta$ holds for any $\theta \in \Theta_{i n}$. Then, for sufficiently large $N, L_{N}(z, \theta)$ takes the minimum in $K_{\epsilon_{0}}(z)$. Next, note that $L_{N}(z, \theta)$ is strictly convex on $\Theta^{\circ}$ (with respect to $\theta$ ) for sufficiently large $N$. Therefore, the equations $\partial_{i} L_{N}(z, \theta)=0$ have a unique solution in $K_{\epsilon_{0}}\left(\theta_{z}\right)$, which is the minimum point. We have

$$
\partial_{i} L_{N}(z, \theta) / N=-z_{i}+\eta_{i}-\partial_{i} \ln \rho(\theta) / N \text {. }
$$

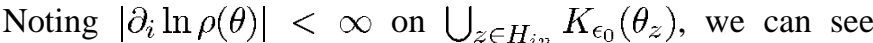
that the solution of the equations $\partial_{i} L_{N}(z, \theta)=0$ equals $z_{i}+\partial_{i} \ln \rho\left(\theta_{z}\right) / N+O\left(1 / N^{2}\right)$. This completes the proof.

Remark: In particular, $f_{m d l}^{w_{J}}=\hat{f}$ holds exactly.

Q.E.D.

We let $w_{\eta}$ denote the uniform prior with respect to $\eta$. Then $w_{\eta}(\theta) d \theta \propto \operatorname{det}\left|g_{i j}\right| d \theta$ holds. Hence, noting (1), we have $\partial_{i} w_{\eta}(\theta)=T_{i j k} g^{j k}$. Therefore, we can see the following lemma holds as the special case of the Lemma 1.

Lemma 2: Suppose Assumptions 1 and 4 hold for $S(\Theta)$ and $w_{\eta}$. For any $N^{\prime} \in N I$

$$
\eta_{i}\left(f_{m d l}^{\eta}\left[x^{N}\right]\right)=\hat{\eta}_{i}+\hat{T}_{i j k} \hat{g}^{j k} / 2 N+O\left(1 / N^{2}\right)
$$

holds uniformly for $x^{\infty} \in T_{N^{\prime}}$.

\section{BIAS-CORRECTED MAXIMUM-LIKELIHOOD ESTIMATOR}

Hereafter, we suppose that $S\left(\Theta_{a}\right)$ is not only minimal but steep. In this case, the expectation of $\hat{\eta}=\bar{x}$ equals $\eta$ itself. Hence, we can say that the MLE is unbiased with respect to the $\eta$-coordinates. Now, we think about the other coordinates $u$. Let $u_{0}$ and $\eta_{0}$ denote the values of $u$ and $\eta$ of the actual distribution, respectively. Thinking of $u$ as the function of $\eta$, taking Taylor expansion of $u$ in the neighborhood of $\eta_{0}$, we 
have

$$
\begin{aligned}
\hat{u}_{i}-u_{0 i}= & \left(\partial^{k} u_{i}\right)_{0}\left(\hat{\eta}_{k}-\eta_{0 k}\right) \\
& +\left(\partial^{l} \partial^{k} u_{i}\right)_{0}\left(\hat{\eta}_{k}-\eta_{0 k}\right)\left(\hat{\eta}_{l}-\eta_{0 l}\right) / 2+O\left(\left|\hat{\eta}-\eta_{0}\right|^{3}\right) .
\end{aligned}
$$

Taking the expectation of both sides, we have

$$
E_{\eta}\left(\hat{u}_{i}-u_{0 i}\right)=\left(\partial^{l} \partial^{k} u_{i}\right)_{0}\left(g_{k l}\right)_{0} / 2 N+O(1 / N \sqrt{N})
$$

Namely, $\hat{u}$ has bias of order $1 / N$. However, if we let

$$
\tilde{u}_{i} \equiv \hat{u}_{i}-\partial^{l} \partial^{k} u_{i}(\hat{\eta}) \hat{g}_{k l} / 2 N
$$

it is known that $\tilde{u}$ 's bias is of order $O\left(N^{-3 / 2}\right)$. Moreover, the mean-square error of $\tilde{u}$ is least (with respect to the $1 / N^{2}$ term) among the efficient estimators (see [1]). The function $f_{b c}^{u}: x^{N} \mapsto p(x \mid \tilde{u}) \in S$ is called the bias-corrected MLE with respect to the coordinate system $u$.

We can prove the following lemma.

Lemma 3: For any $N^{\prime} \in N$,

$$
\eta_{i}\left(f_{b c}^{\theta}\left[x^{N}\right]\right)=\bar{x}_{i}+\hat{T}_{i j k} \hat{g}^{j k} / 2 N+O\left(1 / N^{2}\right)
$$

holds, uniformly for $x^{\infty} \in T_{N^{\prime}}$.

Proof: Plugging in $\theta$ to $u$ of (13), we have

$$
\theta^{i}\left(f_{b c}^{\theta}\left[x^{N}\right]\right)-\theta^{i}\left(\hat{f}\left[x^{N}\right]\right)=-\partial^{l} \hat{g}^{i k} \hat{g}_{k l} / 2 N .
$$

Hence, we have

$$
\begin{aligned}
\eta_{j}\left(f_{b c}^{\theta}\left[x^{N}\right]\right)-\hat{\eta}_{j} & =-\frac{\partial \eta_{j}}{\partial \theta^{i}}\left(\partial^{l} \hat{g}^{i k}\right) \hat{g}_{k l} / 2 N+O\left(1 / N^{2}\right) \\
& =-\hat{g}_{j i}\left(\partial^{l} \hat{g}^{i k}\right) \hat{g}_{k l} / 2 N+O\left(1 / N^{2}\right)
\end{aligned}
$$

Now, differentiating $g_{j i} g^{i k}=\delta_{j}^{k}$ with respect to $\eta_{l}$, we have

$$
-g_{j i} \partial^{l} g^{i k}=\left(\partial^{l} g_{j i}\right) g^{i k}=\frac{\partial g_{j i}}{\partial \theta^{m}} \frac{\partial \theta^{m}}{\partial \eta_{l}} g^{i k}=T_{j m i} g^{m l} g^{i k}
$$

Hence, we have

$$
-g_{j i}\left(\partial^{l} g^{i k}\right) g_{k l}=T_{j m i} g^{m l} g^{i k} g_{k l}=T_{j m i} g^{m l} \delta_{l}^{i}=T_{j m i} g^{m i}
$$

Together with (14), we obtain the claim of the lemma. Q.E.D.

\section{DISCUSSION}

Corollary 1 and Lemmas 2 and 3 give asymptotic forms of $\tilde{f}_{w_{J}}, f_{m d l}^{\eta}$ and $f_{b c}^{\theta}$, and yield the following theorem.

Theorem 2: Let $S(\Theta)$ be a minimal steep exponential family and $\Theta$ satisfy Assumption 1. Suppose that Assumptions 2 and 3 hold for $S(\Theta)$ and $w_{J}$, and that Assumption 4 holds for $S(\Theta)$ and $w_{\eta}$. For any $N^{\prime} \in N I$, the differences between $\eta\left(f_{m d l}^{\eta}\left[x^{N}\right]\right), \eta\left(\tilde{f}_{w_{J}}\left[x^{N}\right]\right)$, and $\eta\left(f_{b c}^{\theta}\left[x^{N}\right]\right)$ are of order $O\left((\ln N)^{1 / 2} N^{-3 / 2}\right)$, uniformly for $x^{\infty} \in T_{N^{\prime}}$.

Since the geometrical fact that $\tilde{f}_{w_{J}}$ is a -1 -projection of $f_{w_{J}}$, we can expect that Theorem 2 represents a certain property of the maximin estimator $f_{w_{J}}$. In particular, we can think that the maximin property has strong relation with unbiasness with respect to the canonical parameter. The canonical parameter has a geometrical interpretation that it is the affine parameter associated with respect to the 1-connection [1]. It would be interesting to analyze the relation between the above optimality and the 1-connection.

Using Theorem 1 and Lemma 1, we can also obtain the following theorem, which is dual to Theorem 2 .
TABLE I

DEPENDENCY OF Estimators ON PRIOR

\begin{tabular}{|c||c|c|c|}
\hline Prior $w d \theta$ & $d \theta$ & $\sqrt{\operatorname{det} \mid g_{i j}} \mid d \theta$ & $d \eta$ \\
\hline$\tilde{f}_{w}$ & $\eta$-unbiased & $\theta$-unbiased & \\
\hline$f_{m d l}^{w}$ & & $\eta$-unbiased & $\theta$-unbiased \\
\hline
\end{tabular}

Theorem 3: Let $S(\Theta)$ be a minimal steep exponential family and $\Theta$ satisfy Assumption 1. Suppose that Assumptions 2 and 3 hold for $S(\Theta)$ and $d \theta$, and that Assumption 4 holds for $S(\Theta)$ and $w_{J}$. For any $N^{\prime} \in N I$, the differences between $\eta\left(f_{m d l}^{w_{J}}\left[x^{N}\right]\right), \eta\left(\tilde{f}_{d \theta}\left[x^{N}\right]\right)$, and $\hat{\eta}$ are of order $O\left(1 / N^{2}\right)$, uniformly for $x^{\infty} \in T_{N^{\prime}}$.

We can illustrate the above two theorems by Table I, where we ignore the terms of order $o(1 / N)$. Note that $\eta$ is the affine parameter with respect to the -1 -connection and is dual to $\theta$. From this table, we assume that $\tilde{f}_{w}$ and $f_{m d l}^{w}$ form a dual pair, because if we exchanged $\eta$ and $\theta$, and the Bayes and MDL, then we would have the same table again.

Now, we give some examples.

Example 1 (Bernoulli Sources): Define $\mathcal{X}=\{0,1\}$

$$
S=\left\{p(x \mid \eta)=\eta^{x}(1-\eta)^{1-x} \mid 0<\eta<1\right\}
$$

and $\theta=\ln (\eta /(1-\eta))$. We have

$$
g(\eta) \equiv g^{11}=\partial \theta / \partial \eta=1 / \eta(1-\eta) .
$$

We let $k \equiv \sum_{t=1}^{N} x_{t}$. As it is well known, the Bayes predictor with Jeffreys prior equals $(k+0.5) /(N+1)$, denoted by $\eta_{L}$. Next, we consider the MDL estimator. The total description length for the MDL with respect to the $\eta$-coordinates is

$$
\begin{aligned}
-k \ln \eta-(N-k) \ln (1-\eta)-(\ln \eta+\ln (1-\eta)) / 2 \\
=-(k+0.5) \ln \eta-(N-k+0.5) \ln (1-\eta) .
\end{aligned}
$$

The value of $\eta$ minimizing the total description length is $\eta_{\text {mdl }}=(k+0.5) /(N+1)$, which strictly equals $\eta_{L}$. Finally, we consider the bias-corrected MLE. Since

$$
\begin{aligned}
T_{111} & =E_{\theta}\left((x-\eta)^{3}\right)=\eta(1-\eta)^{3}-(1-\eta) \eta^{3} \\
& =\eta(1-\eta)(1-2 \eta)
\end{aligned}
$$

holds, we have

$$
\hat{T}_{111} \hat{g}^{11}=1-2 \hat{\eta}=1-2 k / N
$$

Hence, we have

$\eta_{b c}=k / N+(1-(2 k / N)) /(2 N)+O\left(1 / N^{2}\right)=\eta_{L}+O\left(1 / N^{2}\right)$.

Example 2 (Normal Distributions): The family of normal distributions is defined as

$$
\begin{aligned}
S=\{p(z \mid \mu, \sigma)= & (1 / \sqrt{2 \pi} \sigma) \\
\cdot & \left.\exp \left(-(z-\mu)^{2} / 2 \sigma^{2}\right) \mid \mu \in \Re, \sigma^{2}>0\right\} .
\end{aligned}
$$

If we define a new vector valued random variable $x$ as $x_{1}=z, x_{2}=z^{2}$ and let $\theta^{1}=\mu / \sigma^{2}, \theta^{2}=-1 /\left(2 \sigma^{2}\right)$, and

$$
\psi(\theta)=-\left(\theta^{1}\right)^{2} /\left(4 \theta^{2}\right)+(1 / 2) \cdot \ln \left(-\pi / \theta^{2}\right)
$$

we can see $S$ is an exponential family. Now, we have

$$
\operatorname{det}\left|g_{i j}\right|=-2 /\left(\theta^{2}\right)^{3}=16 \sigma^{6} \text {. }
$$


In reference to estimation of $\mu$, both the projected Bayes estimator and the MDL estimator give the same result as the MLE. Therefore, it is sufficient to consider estimation of a coordinate $\sigma^{2}$ alone. We describe just the result for simplicity. Let $\tilde{\sigma}^{2}$ and $\hat{\sigma}^{2}$ mdl denote the projected Bayes estimate (with $w_{J}$ ) and the MDL estimate (with $w_{\eta}$ ) of $\sigma^{2}$, respectively, then we have $\tilde{\sigma}^{2}=(N+1) v^{2} /(N-2)$ and $\hat{\sigma}^{2} m d l=N v^{2} /(N-3)$, where $v^{2}=\bar{z}^{2}-\bar{z}^{2}$. The difference between the two is of order $O\left(1 / N^{2}\right)$. Compare this with the so-called unbiased variance $N v^{2} /(N-1)$.

In the above examples, we were able to analytically obtain the projected Bayes estimator and the MDL estimator. In general, however, it is difficult to do so. In such cases, Theorem 1 and Lemma 1 provide us with a way to approximate the projected Bayes estimator and the MDL estimator. Actually in [14], a similar method as the proof of Theorem 1 is used to approximate the Bayes codes for the Markov sources.

\section{APPENDIX}

PROOF OF PROPOSITION 2

Suppose first that $\xi(\theta) F_{N_{0}}\left(z_{0}, \theta\right)$ is integrable on $\Theta$, where $z_{0} \in H_{\text {in }}$ holds, and $N_{0}$ and $C$ are positive reals. In the sequel, we assume that $z \neq z_{0}$, since $\xi(\theta) F_{N}\left(z_{0}, \theta\right)$ is integrable when $N \geq N_{0}$.

Let $H^{\prime}$ denote a compact set of $\Re^{n}$ such that $H_{i n} \subset H^{\prime \circ}$ and $H^{\prime} \subset H_{a}^{\circ}$ hold. (There exists such a set, since $H_{\text {in }} \subset H_{a}^{\circ}$.) Let $\theta_{z}$ denote the value of $\theta$ which corresponds to $\eta=z$. Then, we have $F_{1}(z, \theta) \leq F_{1}\left(z, \theta_{z}\right)$. Moreover, the function $z \mapsto F_{1}\left(z, \theta_{z}\right)$ is continuous on $H_{a}^{\circ}$. Hence, $F_{1}\left(z, \theta_{z}\right)$ is bounded on $H^{\prime}$, i.e., we have $F_{1}(z, \theta) \leq C_{1}$ for $z \in H^{\prime}$ and $\theta \in \Theta_{a}$. Define a function $\lambda:\left(H_{i n}-\left\{z_{0}\right\}\right) \rightarrow \Re^{+}$as

$$
\lambda(z) \equiv \max \left\{\beta \mid z+\beta \cdot\left(z-z_{0}\right) \in H^{\prime}\right\} .
$$

Let

$$
d_{1}=\max _{x, y \subset H_{i n}}|x-y|
$$

and

$$
d_{2}=\inf _{x \in \partial H_{i n}, y \in \partial H^{\prime}}|x-y| .
$$

( $d_{2} \neq 0$ by the definition of $H^{\prime}$, and $d_{1} \neq 0$ holds.) Then, $\lambda(z)\left|z-z_{0}\right| \geq d_{2}$ holds, i.e., we have $\lambda(z) \geq d_{2} / d_{1}$. Let $z^{\prime}$ denote $z+\lambda(z)\left(z-z_{0}\right)$, then $z^{\prime} \in H^{\prime}$ and

$$
z=\left(\lambda(z) z_{0}+z^{\prime}\right) /(1+\lambda(z))
$$

hold. Since $z^{\prime} \in H^{\prime}$, we have $F_{1}\left(z^{\prime}, \theta\right) \leq C_{1}$ for $\theta \in \Theta_{a}$. Therefore, we have

$$
\begin{aligned}
F_{1}(z, \theta) & =F_{1}\left(z_{0}, \theta\right)^{\lambda(z) /(1+\lambda(z))} F_{1}\left(z^{\prime}, \theta\right)^{1 /(1+\lambda(z))} \\
& \leq F_{1}\left(z_{0}, \theta\right)^{\lambda(z) /(1+\lambda(z))} C_{1}^{1 /(1+\lambda(z))} .
\end{aligned}
$$

Hence, we have

$$
F_{1}(z, \theta)^{N_{0}(1+\lambda(z)) / \lambda(z)} \leq F_{N_{0}}\left(z_{0}, \theta\right) C_{1}^{N_{0} / \lambda(z)} .
$$

Let

$$
N_{1} \equiv \sup _{z} N_{0}(1+\lambda(z)) / \lambda(z)(<\infty)
$$

and $u(z) \equiv N_{1}-N_{0}(1+\lambda(z)) / \lambda(z)$. Since $u(z) \geq 0$ and $F_{1}(z, \theta) \leq C_{1}$ hold, we have $F_{1}(z, \theta)^{u(z)} \leq C_{1}^{u(z)}$.
Hence, we obtain

$$
\begin{aligned}
F_{N_{1}}(z, \theta) & =F_{1}(z, \theta)^{N_{1}} \\
& =F_{1}(z, \theta)^{N_{0}(1+\lambda(z)) / \lambda(z)} \cdot F_{1}(z, \theta)^{u(z)} \\
& \leq F_{N_{0}}\left(z_{0}, \theta\right) C_{1}^{N_{0} / \lambda(z)} \cdot C_{1}^{u(z)} .
\end{aligned}
$$

Since $F_{v}(z, \theta) \leq C_{1}^{v}$ hold for $v \geq 0$, we have

$$
F_{N_{1}+v}(z, \theta) \leq F_{N_{0}}\left(z_{0}, \theta\right) C_{1}^{N_{0} / \lambda(z)} C_{1}^{u(z)+v} .
$$

Noting that $0<N_{0} / \lambda(z)<\infty$ and $0 \leq u(z) \leq N_{1}$ holds for $z \in Z_{\text {in }}-\left\{z_{0}\right\}$, we have

$$
F_{N_{1}+v}(z, \theta) \leq F_{N_{0}}\left(z_{0}, \theta\right) C_{v}
$$

where $C_{v}$ is a real determined by $v$. Since $\xi(\theta) F_{N_{0}}\left(z_{0}, \theta\right)$ is integrable on $\Theta, \xi(\theta) F_{N_{1}+v}(z, \theta)$ is integrable on $\Theta$ for any $v>0$ and any $z \in H_{\text {in }}$. Since the converse is trivial, this implies the proposition.

Q.E.D.

\section{ACKNOWLEDGMENT}

The author wishes to express his sincere gratitude to the following people: H. Nagaoka, T. Kawabata, N. Abe, T. Okamura, Y. Kubo, A. R. Barron, K. Nakamura, and T. Fujita.

\section{REFERENCES}

[1] S.-I. Amari, Differential-Geometrical Methods in Statistics 2nd ed. (Lecture Notes in Statistics, vol. 28). Berlin, Germany: SpringerVerlag, 1990.

[2] A. R. Barron, "Logically smooth density estimation," Ph.D. dissertation, Dept. Elec. Eng., Stanford Univ., Stanford, CA, 1985.

[3] A. R. Barron and T. M. Cover, "Minimum complexity density estimation," IEEE Trans. Inform. Theory, vol. 37, pp. 1034-1054, 1991.

[4] J. M. Bernardo, "Reference posterior distributions for Bayesian inference," J. Roy. Statist. Soc. B., vol. 41. pp. 113-147, 1979.

[5] L. Brown, Fundamentals of Statistical Exponential Families. Inst Math. Statist., 1986

[6] B. Clarke and A. R. Barron, "Information-theoretic asymptotics of Bayes methods," IEEE Trans. Inform. Theory, vol. 36, pp. 453-471, May 1990.

[7] _ "Jeffreys prior is asymptotically least favorable under entropy risk," J. Statist. Planning and Inference, vol. 41, pp. 37-60, 1994.

[8] L. D. Davisson, "Universal noiseless coding," IEEE Trans. Inform. Theory, vol. IT-19, pp. 783-795, Nov. 1973.

[9] L. D. Davisson and A. Leon-Garcia, "A source matching approach to finding minimax codes," IEEE Trans. Inform. Theory, vol. IT-26, pp. 166-174, Mar. 1980

[10] H. Jeffreys, Theory of Probability, 3rd ed. Berkeley, CA: Univ. of California Press, 1961.

[11] T. Matsushima, H. Inazumi, and S. Hirasawa, "A class of distortionless codes designed by Bayes decision theory," IEEE Trans. Inform. Theory, vol. 37, pp. 1288-1293, 1991.

[12] J. Rissanen, "Modeling by shortest data description," Automatica, vol. 14, pp. 465-471, 1978.

[13] _ , "Stochastic complexity," J. Roy. Statist. Soc. B, vol. 49, pp 223-239 and 252-265, 1987.

[14] J. Takeuchi and T. Kawabata, "Approximation of Bayes code for Markov sources," in Proc. 1995 IEEE Int. Symp. on Information Theory, 1995 p. 391.

[15] C. S. Wallace and D. M. Boulton, "An invariant Bayes method for point estimation," Classification Soc. Bull., vol. 3, pp. 11-34, 1975.

[16] C. S. Wallace and P. R. Freeman, "Estimating and inference by compact coding," J. Roy. Statist. Soc. B, vol. 49. no. 3 pp. 240-265, 1987.

[17] Q. Xie and A. R. Barron, "Minimax redundancy for the class of memoryless sources,"IEEE Trans. Inform. Theory, to be published.

[18] K. Yamanishi, "A learning criterion for stochastic rules," Mach. Learn. (Special Issue on COLT '90), vol. 9, no. 2/3, pp. 165-203, 1992.

[19] J. Takeuchi, "On the convergence rate of the MDL estimator with respect to the KL-divergence" (in Japanese), in Proc. 16th Symp. on Information Theory and its Application, 1993, pp. 161-164.

[20] J. Takeuchi and N. Abe, "Evaluation of Laplace-like estimators in the probabilistic PAC learning model" (in Japanese), IEICE Tech. Rep. IT92-128, 1993-03. pp. 1-6, 1993. 